\title{
Spontaneous Remission of Severe Systemic Langerhans Cell Histiocytosis with Bladder Involvement: A Case Study
}

\author{
Isotta M. Magaton ${ }^{a} \quad$ Alexandar Tzankov ${ }^{b} \quad$ Fatime Krasniqi ${ }^{c}$ \\ Christof Rottenburger $^{d}$ Rosanna Zanetti-Daellenbach ${ }^{\mathrm{e}}$ \\ Peter Grendelmeier ${ }^{f} \quad$ Viola Heinzelmann-Schwarz ${ }^{g} \quad$ Michael Mayr $^{h}$ \\ Fabienne D. Schwab ${ }^{9}$

\begin{abstract}
a Hospital for Women, Clinic Fontana, Cantonal Hospital Graubünden, Chur, Switzerland; ${ }^{b}$ Institute of Pathology, University Hospital Basel, Basel, Switzerland; ' ${ }^{\mathrm{C}}$ Department of Oncology, University Hospital Basel, Basel, Switzerland; ${ }^{d}$ Clinic of Radiology and Nuclear Medicine, University Hospital Basel, Basel, Switzerland; ${ }^{e}$ Department of Gynecology and Gynecological Oncology, Claraspital, Basel, Switzerland; 'Department of Pulmonology, University Clinic of Internal Medicine, Cantonal Hospital Baselland, Liestal, Switzerland; ${ }^{9}$ Department of Gynecology and Gynecological Oncology, Hospital for Women, University Hospital Basel, Basel, Switzerland; ' $M$ Medical Outpatient Clinic, University Hospital Basel, Basel, Switzerland
\end{abstract}

\section{Keywords}

Alkaline phosphatase · Bladder · CD1a-positive histiocytes · Cervical cancer · Langerhans cell histiocytosis · Langerin · Multisystem disease · Remission · Risk organs · Therapy

\begin{abstract}
Background: The clinical presentation of Langerhans cell histiocytosis (LCH) is heterogeneous ranging from single-organ involvement to systemic disease causing substantial morbidity and mortality. We describe an unusual course of severe multisystem LCH with spontaneous remission. Case Presentation: We report on a 45-year-old Caucasian woman with cervical cancer, FIGO stage IVB. Five months after the end of combined radiochemotherapy and brachytherapy, the patient was readmitted because of severe dysuria. Sterile leukocyturia
\end{abstract}




\section{Case Reports in Oncology}

was seen, and cystoscopy revealed only 3 unspecific small mucosal lesions compatible with postradiation cystitis. Incidentally, a computed tomography (CT) scan of the body showed diffuse micronodular and cystic lesions in lungs and hypodense lesions in the liver. Biopsies revealed infiltrations of CD1a and Langerin (CD207)-positive histiocytes in the lung, liver, and bladder. Additionally, positron emission tomography-CT (PET-CT) was compatible with bone involvement. Retrospective analysis revealed that the increase in alkaline phosphatase might have been a surrogate of bone marrow infiltration with osseous activity. Repeated pneumothoraces occurred, and only one course of vinblastine-prednisolone could be applied. Despite ongoing tobacco consumption and without further therapy, PET-CT showed considerable remission 2 months later. However, despite stable remission, documented by serial PET and conventional CT scans, persistent infiltration of the bladder by Langerhans histiocytes could still be demonstrated 17 months later. Unfortunately, cervical cancer recurred and progressed. Conclusion: Multisystem LCH may rapidly occur, may be oligosymptomatic and, even in high-risk cases, remission without specific therapy might occur. Whether alkaline phosphatase might be a surrogate to monitor osseous disease activity has to be further explored.

(c) 2017 The Author(s)

Published by S. Karger AG, Basel

\section{Background}

Langerhans cell histiocytosis (LCH), previously known as histiocytosis $\mathrm{X}$, is a rare disease mostly affecting children. For adults, the annual prevalence of LCH can only be estimated and is about 1 case per 560,000 adults [1]. Most of the knowledge about diagnosis and therapy derives from pediatric studies [2,3].

LCH is characterized by clonal proliferation of pathologic cells with characteristics of histiocytes with varying proportions of macrophages, T-lymphocytes, eosinophils, plasma cells, and multinucleated giant cells accumulating and forming characteristic granuloma-like lesions in various organs [4]. The main diagnostic feature is morphologic identification of the characteristic Langerhans cells. Additionally, positive staining of the lesional cells with CD1a and langerin (CD207) is required for definitive diagnosis. It has been demonstrated that the expression of Langerin strongly correlates with the presence of cytoplasmic Birbeck granules, which can be seen on electron microscopy $[2,4]$.

The clinical presentation is very heterogeneous ranging from a single organ or system involvement, generally benign, to a life-threatening multisystem disease [4]. Virtually every organ can be involved. Specific data about organ involvement in adults are sparse; the organs most frequently involved in children and adolescents are skeleton ( $80 \%$ of cases), skin (33\%), and the pituitary gland (25\%). Other organs involved are the liver, spleen, the hematopoietic system and the lungs (15\% each), lymph nodes (5-10\%), and the central nervous system (2-4\%, excluding the pituitary) [5]. Patients with disease limited to one organ, usually bones, skin, lungs or lymph nodes, also called "single-system" or "low-risk disease," have a good prognosis and usually need minimal or in some cases no treatment. In contrast, multisystem disease with risk organ involvement, defined as infiltration of bone marrow, liver, spleen, and/or central nervous system, is associated with a less favorable prognosis with increased risk of mortality (10-20\%) and morbidity [3, 6]. In a mixed cohort of children and adults of the Mayo Clinic, 30\% had multisystem and 70\% single-system disease. In cases with single-system disease, bones were most often involved (52\%), followed by pulmonary (39\%), mucocutaneous (6.5\%), thalamic (1\%), and lymphatic LCH (0.5\%). Predicting factors for progressive LCH were multisystem LCH with bone or pituitary-thalamic axis involve- 
ment, multisystem LCH in 3 or more organs, single osseous LCH in 3 or more bones, singlesystem LCH of mucous membranes, young age ( $<5$ years), and hepatosplenomegaly in patients at the age of 3 years or less [7].

The immune mechanisms underlying $\mathrm{LCH}$ are still not fully understood [4]. Cancerassociated mutations (BRAF V600E in 50\% of cases, MAP2K1 in $30 \%$ of cases and MAP3K1 mutations in $10 \%$ of cases) were identified $[3,8,9]$, and clonality was shown in all forms of $\mathrm{LCH}$, except for smoking-associated adult pulmonary $\mathrm{LCH}$, indicating that $\mathrm{LCH}$ might rather be neoplastic with variable behavior than a reactive disorder triggered by infections or malignancies [3, 4].

We describe a case of multisystemic LCH with involvement of the bladder, which is extremely unusual. Despite risk organ involvement, considerable regression occurred and symptoms disappeared without specific therapy.

\section{Case Presentation}

A 45-year-old Caucasian woman was diagnosed with stage IVB cervical cancer (Fig. 1). Obstruction of the right kidney was seen, and a ureter catheter was inserted. The cancer was treated with 6 courses of combined radiochemotherapy followed by brachytherapy. The patient's past medical history did not reveal any previous cancer manifestation. She had been smoking for several years, cumulative 45 pack-years. She abused cocaine and heroin, but definitively stopped drug abuse a long time ago. Regarding previous surgical procedures, the patient had a gastric banding, abdominoplasty, and recurrent abscess excision in the breast due to mastitis non-puerperalis. She reported spontaneous vaginal deliveries when she was 18 and 20 years old.

Side-effects of radiation-chemotherapy were transient local irritations with dysuria and pollakisuria. Additionally, one pyelonephritis episode occurred 3 months after the end of therapy while ureter catheter was in situ. Eight months after cancer diagnosis and 5 months after the end of the radiation-chemotherapy, the patient complained again of heavy dysuria, pollakisuria, and additionally lower-abdominal cramps (Fig. 1). Urinary tract infections were assumed but could not be proven microbiologically. Empiric therapy with different antibiotics was not successful in pain relief. Therefore, the differential diagnosis of overactive bladder syndrome and postradiation irritation was made. Analgesic and spasmolytic therapy with oxycodon, morphine, and trospium chloride were not sufficient to control the complaints, and the patient was admitted for further diagnostic evaluation. Sterile leukocyturia was seen, and cystoscopy revealed 3 unspecific small mucosal lesions and one ulceration compatible with postradiation cystitis. To exclude a relapse of her cervical cancer, computed tomography (CT) of the thorax, abdominal wall, and pelvis was performed. Surprisingly, multiple micronodular and cystic lesions in both lungs and diffuse hypodense lesions in the liver were detected. ELISPOT assay for Mycobacterium tuberculosis, HIV screening test, and ANA/ANCA were negative. Biopsies of the liver and the lung revealed infiltrations of Langerin (CD207) and CD1a-positive histiocytes admixed with eosinophils in both organs, and LCH was diagnosed (Fig. 1 and Fig. 2a, b). Sequencing of BRAF and MAP2K1 revealed wild-type genes. At this time, LCH of the bladder was suspected and also confirmed by biopsy (Fig. 2c, d). On this occasion, the ureter-catheter could be removed. To complete the staging for LCH, a whole-body positron emission tomography-CT (PET-CT) was performed, and the final diagnosis of multisystem LCH involving lungs, liver, bone, and the bladder was established (Fig. 1 and Fig. 3a-d). Interestingly, neither clinical symptoms nor lab values re- 


\section{Case Reports in Oncology}

flected the severe involvement of lungs, liver, and bone marrow seen on PET-CT. However, retrospective analysis revealed that an increase of alkaline phosphatase might have been a surrogate of bone marrow infiltration with osseous disease activity (Fig. 1). Lung function performed after diagnosis showed capacity at the lower limit of normal (86\% of predicted value), severely impaired diffusion capacity ( $48 \%$ of predicted value) as well as slight hypoxemia $\left(\mathrm{pO}_{2}\right.$ of $\left.63 \mathrm{~mm} \mathrm{Hg}\right)$ despite hyperventilation $\left(\mathrm{pCO}_{2}\right.$ of $\left.32 \mathrm{~mm} \mathrm{Hg}\right)$. However, the patient did not suffer from cough or dyspnea.

The patient was advised to quit tobacco use. However, despite nicotine-replacement therapy and varenicline, the patient was not able to stop smoking. Due to the multisystem presentation with risk organ involvement (lung, liver, and bone), therapy with vinblastine $\left(6 \mathrm{mg} / \mathrm{m}^{2}\right.$ i.v. day 1) and steroids ( $40 \mathrm{mg} / \mathrm{m}^{2}$ daily) was started (Fig. 1). However, 3 days later the patient suffered from a right-sided pneumothorax. Resection of bullous cysts and talc-pleurodesis were performed. In order not to jeopardize the healing process, vinblastine and steroids were discontinued. During the next weeks, the patient experienced 2 more pneumothoraces (one on the left side and one recurrence on the right side) in need of further interventions with pleurodesis and wedge resection of the bullous cysts (Fig. 1).

Surprisingly, despite therapy discontinuation, the patient became asymptomatic, and two months later a follow-up PET-CT showed remarkable partial remission of LCH (Fig. 1). However, despite stable remission, documented by serial PET-CT and conventional CT scans (Fig. 1 and Fig. 3e-g), persistent infiltration of the bladder by LCH could be demonstrated at the last follow-up 17 months after diagnosis. Unfortunately, cervical cancer recurred, and laparoscopic evaluation showed a noncurative condition with positive pelvic lymph nodes (Fig. 1 and Fig. 4).

\section{Discussion}

We describe a case of multisystem LCH with risk organ involvement. There are several teaching points that make our case particularly interesting. First, the involvement of the urogenital tract by LCH is a rarity. There are only few reports in the literature, which describe such an involvement. Besides the bladder, infiltration of the kidneys and the testes has been described [10]. In contrast, genital and oral mucosa is the most common mucous infiltration in cases of mucocutaneous LCH [7]. In our case, there was no mucocutaneous manifestation of LCH. Dysuria, pollakisuria and lower abdominal cramps were the leading symptoms, initially misjudged as infection, overactive bladder syndrome, and postradiation cystitis. Second, because the patient was asymptomatic, LCH was missed and only diagnosed by incidental findings on CT scans. In spite of extended lesions in the liver, lungs, and bone marrow, there were no specific symptoms either clinically, or in the laboratory, which could suggest manifestations in these organs. Interestingly, a transient increase in the alkaline phosphatase was retrospectively detected. We assume that this increase was caused by osseous disease activity in the context of bone marrow infiltration. Whether alkaline phosphatase could be a useful marker in patients with osseous LCH has to be further explored. In the literature, around $20 \%$ of adults with pulmonary $\mathrm{LCH}$, isolated or as part of multisystem disease, have no symptoms [11]. Our case confirms that even extended multisystemic LCH can evolve subclinically, and symptoms of specific organ involvement can be misleading. Third, it is thought that the beginning of the disease process may precede symptoms by several years [7]. The median delay from first symptoms to diagnosis is around 1 year; however, it is shorter in patients with mucocutaneous, thalamic, and lymphatic manifestations [7]. 


\section{Case Reports in Oncology}

Further, pneumothoraces caused by pulmonary LCH and liver manifestation are judged to be indicators for advanced and late manifestations of LCH [7]. In our case, 9 months before, a PET-CT scan was performed for cancer staging and revealed no signs of LCH. Therefore, our case demonstrates that even extended multisystem disease may evolve very fast and may occur within few months. Fourth, multisystem LCH can be associated with other malignancies. In a retrospective study of a mixed population of children and adults with biopsyproven LCH (median follow-up 4 years, range 1-47.5 years), 27 out of 314 (8.5\%) had a coexisting neoplasm [7]. LCH may precede, occur currently, or occur after the malignancy [12]. The causality between LCH and the occurrence of a second malignancy has still to be determined on an individual basis. A key part of the classification of LCH as a neoplasm has been the identification of $B R A F \mathrm{~V} 600 \mathrm{E}$ (B-Raf proto-oncogene, serine/threonine kinase) gene mutations in 35-60\% of cases [13]. In our patient, gene sequencing of BRAF and MAP2K1 revealed wild-type genes, rather supporting the hypothesis of an immune-reactive disorder and not a neoplastic disorder. There is some evidence that immature dysregulation leads to an aberrant reaction between Langerhans cells and T lymphocytes. This reaction could be triggered by various stimuli including viruses or malignancies [4]. Our patient developed LCH after the diagnosis of advanced cervical cancer, which was caused by Human Papilloma Virus 16. Additionally, the affection of the bladder by LCH corresponded to the area of radiotherapy. Due to the anatomical proximity of the bladder and radiation field, one may postulate that radiotherapy was a trigger for Langerhans cell activation. Further, the patient is a smoker and smoking is a strong risk factor for pulmonary LCH involvement. Taken together, in our patient there are some potential triggers which may have led to an immune-reactive dysregulation of histiocytes and the evolution of LCH. Fifth, multisystem LCH with risk-organ involvement is associated with a poor prognosis, and experts recommend chemotherapy as treatment $[2,7,11]$. In our patient, the clinical course was astonishing. Shortly after diagnosis, the pulmonary situation worsened and the patient suffered three pneumothoraces. In the following, impressive partial remission occurred, followed by stable disease until the last follow-up 17 months after diagnosis. The role of cigarette smoking in pulmonary LCH appears very convincing, and cessation of smoking is recommended for regression [14, 15]. Surprisingly, the patient remained asymptomatic regarding $\mathrm{LCH}$ although smoking cessation could not be achieved, cervical cancer could not be cured and even progressed, and therapy with vinblastine and steroids was discontinued after the first course.

\section{Conclusion}

We described a rare case of adult multisystem LCH with unusual presentation and course. In this case, heavy dysuria mimicking urinary tract infection was the first symptom and not dyspnea despite advanced pulmonary LCH. Physicians should be aware of unusual organ manifestations and oligo-symptomatic presentation of LCH. Further, the case demonstrates that even in severe LCH with risk organ involvement partial remission and stabilization may occur without systemic therapy. Whether alkaline phosphate might be a useful marker to monitor osseous disease activity has to be explored in further studies. 
Magaton et al.: Spontaneous Remission of Severe Systemic Langerhans Cell Histiocytosis with Bladder Involvement: A Case Study

\section{Statement of Ethics}

Hereby, we confirm that the patient has given her written consent for the case report to be published.

\section{Disclosure Statement}

All authors declare that they have no competing interests. The case study was not funded.

\section{Author Contributions}

Herein, we declare that all authors have made substantial contributions to the case study. I.M.M. drafted the manuscript, A.T. carried out the histopathology and revised the manuscript for important intellectual content, F.K. revised the oncological data, C.R. carried out the radiological studies, P.G. carried out the pneumological studies, V.H.S. revised the manuscript, M.M. and F.D.S. designed the study, have been involved in drafting the manuscript and in final approval. All authors read and approved the final manuscript.

\section{References}

1 Aricò M, Girschikofsky M, Généreau T, Klersy C, McClain K, Grois N, Emile JF, Lukina E, De Juli E, Danesino C: Langerhans cell histiocytosis in adults. Report from the International Registry of the Histiocyte Society. Eur J Cancer 2003;39:2341-2348.

2 Minkov M, Grois N, McClain K, Nanduri V, Rodriguez-Galindo C, Simonitsch-Klupp I, Visser J, Weitzman S, Whitlock J, Windebank K: Langerhans Cell Histiocytosis: Histiocyte Society Evaluation and Treatment Guidelines. April 2009. www.histiocytesociety.org

3 Girschikofsky M, Arico M, Castillo D, Chu A, Doberauer C, Fichter J, Haroche J, Kaltsas GA, Makras P, Marzano AV, de Menthon M, Micke O, Passoni E, Seegenschmiedt HM, Tazi A, McClain KL: Management of adult patients with Langerhans cell histiocytosis: recommendations from an expert panel on behalf of Euro-Histio-Net. Orphanet J Rare Dis 2013;8:72.

4 Abla O, Egeler RM, Weitzman S: Langerhans cell histiocytosis: current concepts and treatments. Cancer Treat Rev 2010;36:354-359.

5 Haupt R, Minkov M, Astigarraga I, Schäfer E, Nanduri V, Jubran R, Egeler RM, Janka G, Micic D, Rodriguez-Galindo C, Van Gool S, Visser J, Weitzman S, Donadieu J; Euro Histio Network: Langerhans cell histiocytosis (LCH): guidelines for diagnosis, clinical work-up, and treatment for patients till the age of 18 years. Pediatr Blood Cancer 2013;60:175-84.

-6 Pierro J, Vaiselbuh SR: Adult Langerhans cell histiocytosis as a diagnostic pitfall. J Clin Oncol 2016;34:e41-e45.

7 Numakura S, Morikawa T, Ushiku T, Toyoshima T, Fukayama M: Langerhans cell histiocytosis of the urinary bladder in a patient with bladder cancer previously treated with intravesical Bacillus CalmetteGuérin therapy. Pathol Res Pract 2014;210:123-126.

-8 Howarth DM, Gilchrist GS, Mullan BP, Wiseman GA, Edmonson JH, Schomberg PJ: Langerhans cell histiocytosis: diagnosis, natural history, management, and outcome. Cancer 1999;85:2278-2290.

-9 Brown NA, Furtado LV, Betz BL, Kiel MJ, Weigelin HC, Lim MS, Elenitoba-Johnson KS: High prevalence of somatic MAP2K1 mutations in BRAF V600E-negative Langerhans cell histiocytosis. Blood 2014;124:1655-1658.

10 Nelson DS, van Halteren A, Quispel WT, van den Bos C, Bovée JV, Patel B, Badalian-Very G, van Hummelen P, Ducar M, Lin L, MacConaill LE, Egeler RM, Rollins BJ: MAP2K1 and MAP3K1 mutations in Langerhans cell histiocytosis. Genes Chromosomes Cancer 2015;54:361-368.

11 Histiocytosis Association: www.histio.org.

-12 Egeler RM, Neglia JP, Puccetti DM, Brennan CA, Nesbit ME: Association of Langerhans cell histiocytosis with malignant neoplasms. Cancer 1993;71:865-873. 


\section{Case Reports in Oncology}

Magaton et al.: Spontaneous Remission of Severe Systemic Langerhans Cell Histiocytosis with Bladder Involvement: A Case Study

\footnotetext{
13 Arceci RJ: Biological and therapeutic implications of the BRAF pathway in histiocytic disorders. Am Soc Clin Oncol Educ Book 2014:e441-e445.

14 Elia D, Torre O, Cassandro R, Caminati A, Harari S: Pulmonary Langerhans cell histiocytosis: a comprehensive analysis of 40 patients and literature review. Eur J Intern Med 2015;26:351-356.

15 Wei P, Lu HW, Jiang S, Fan LC, Li HP, Xu JF: Pulmonary Langerhans cell histiocytosis: case series and literature review. Medicine (Baltimore) 2014;93:e141.
}

Michael Mayr and Fabienne D. Schwab contributed equally to this work.

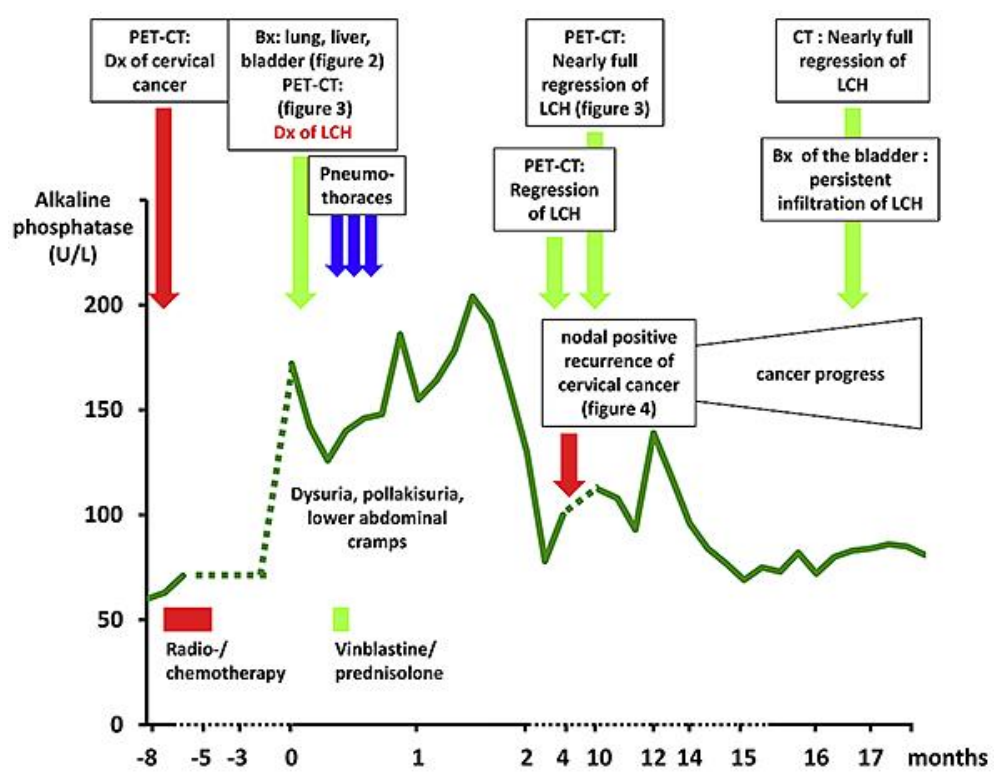

Fig. 1. Disease course of Langerhans cell histiocytosis (LCH). Green line: Values of the alkaline phosphatase (green dotted line: missing values); $x$ axis: follow-up in months (dotted line: compressed time axis); PET$\mathrm{CT}$, positron emission tomography-computed tomography; Dx, diagnosis; Bx, biopsy. 


\section{Case Reports in Oncology}

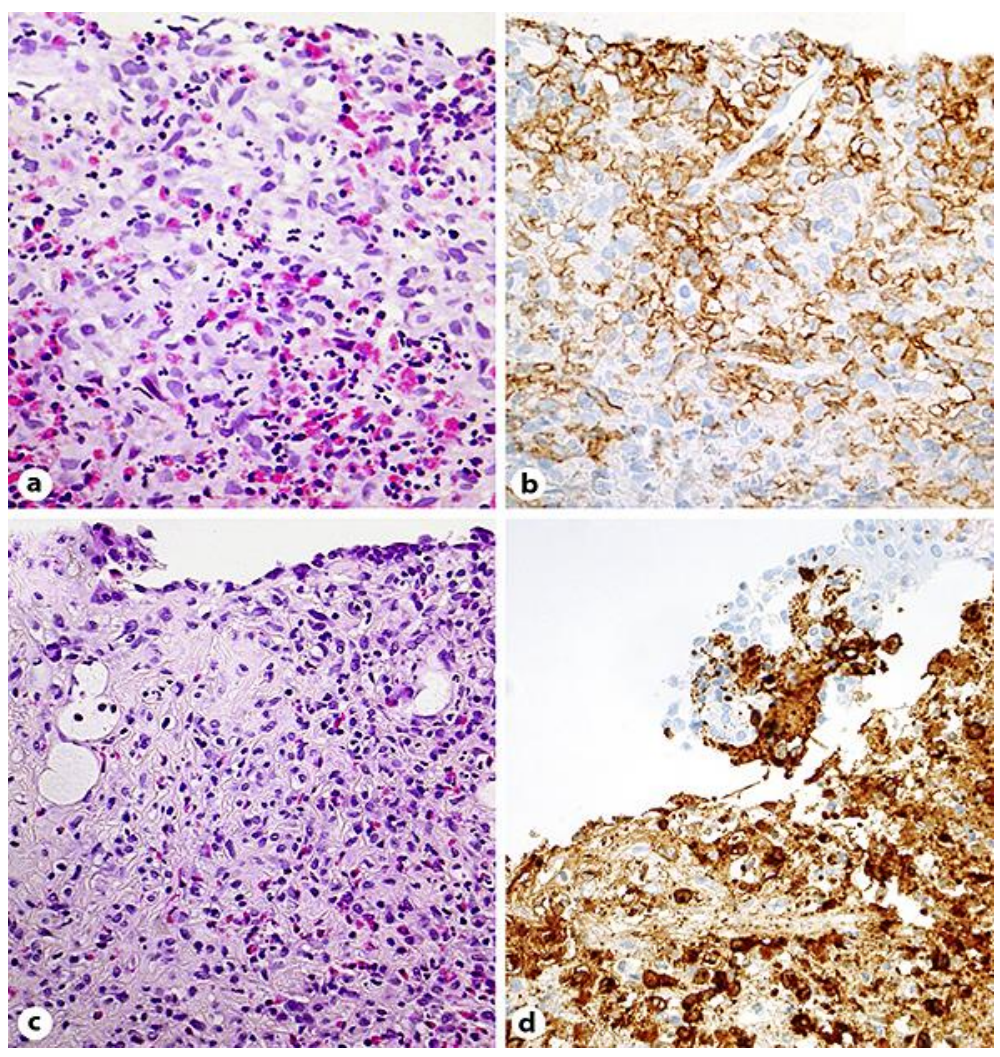

Fig. 2. a Langerhans cell histiocytosis infiltrating the lung. HE. $\times 160$. Note histiocytic cells with cleaved nuclei and broad amphophilic cytoplasms intermingled with eosinophilic granulocytes. $\mathbf{b}$ Expression of langerin in the pulmonary infiltrates. Immunoperoxidase staining. $\times 160$. c Langerhans cell histiocytosis infiltrating the urinary bladder. HE. $\times 100$. Note the partially desquamated urothelial surface and the eosinophilia. $\mathbf{d}$ Expression of CD1a in the bladder infiltrates. Immunoperoxidase staining. $\times 160$. Note the negatively staining urothelium. 


\section{Case Reports in Oncology}
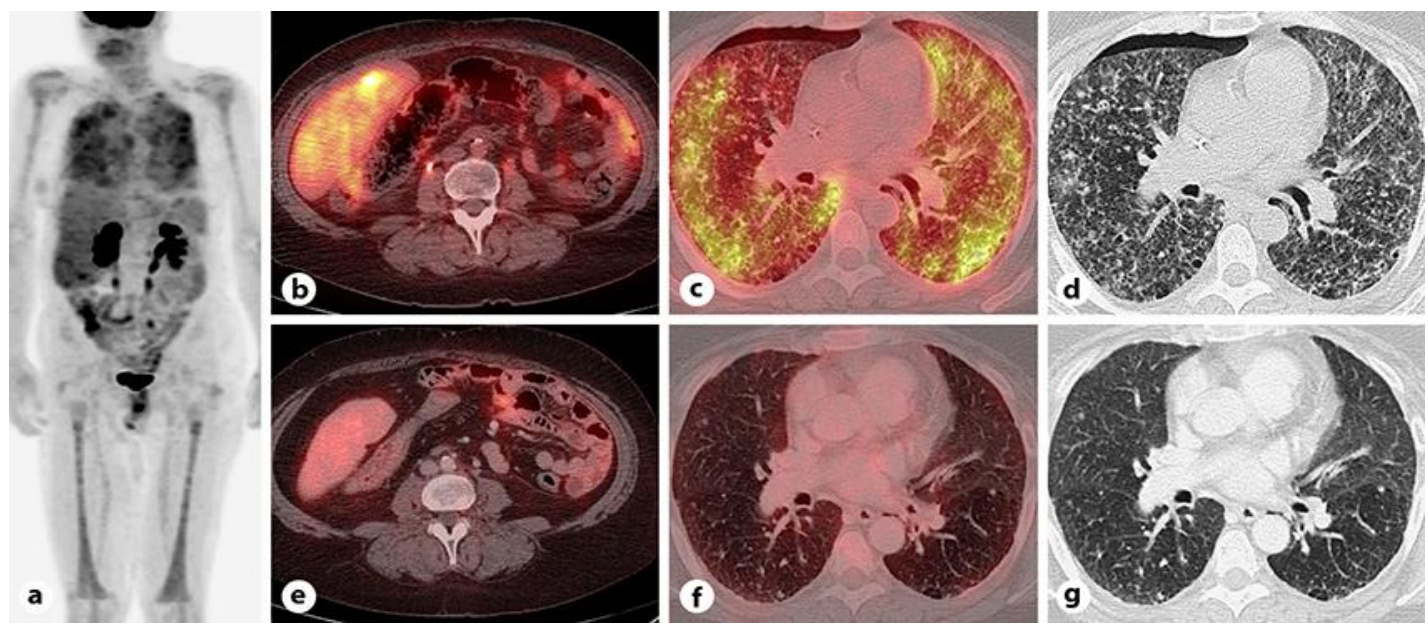

Fig. 3. a Intense, diffuse FDG accumulation in the lungs and liver, and increased accumulation in the bone marrow of both femora as a typical finding for Langerhans cell histiocytosis (maximum intensity projection images of ${ }^{18}$ F-FDG PET/CT). b Hypermetabolic hepatic lesions (fused axial PET/CT slices). c Diffuse hypermetabolism in both lungs (fused axial PET/CT slices). $\mathbf{d}$ Multiple small cystic and micronodular lesions in the lungs, ground glass opacities and small pneumothorax on the right-side ventral view. e Regression of the hypermetabolic lesions in the liver (fused axial PET/CT slices). $\mathbf{f}$ Regression of the hypermetabolism in the lungs (fused axial PET/CT slices). g Nearly complete regression of the cystic and micronodular lesions, the ground glass opacities and the pneumothorax (axial CT).

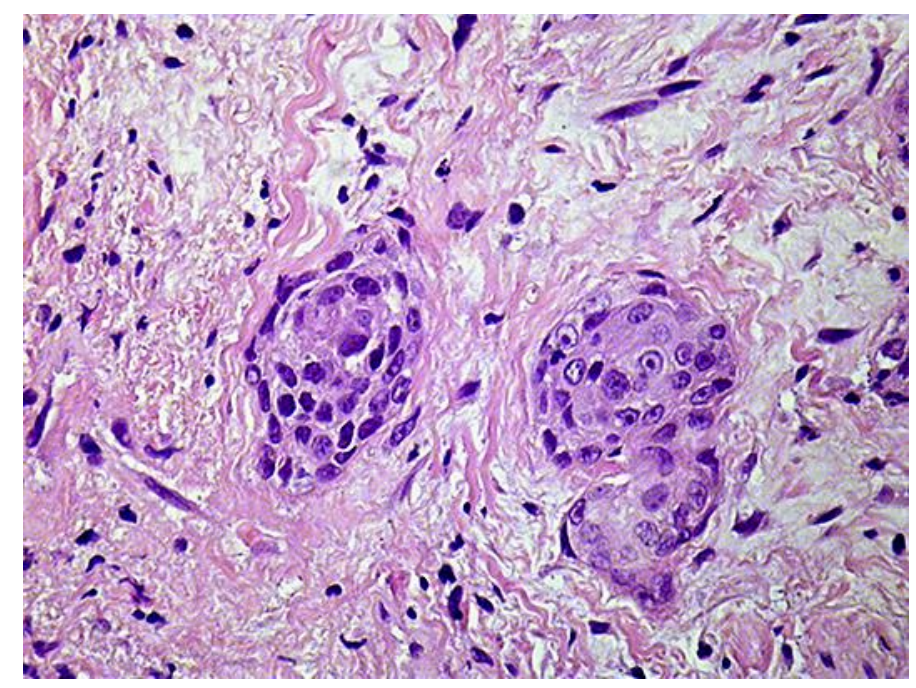

Fig. 4. Invasive endocervical squamous cell carcinoma. HE stain. $\times 360$. 